\title{
Ectopic expression of B and T lymphocyte attenuator in gastric cancer: A potential independent prognostic factor in patients with gastric cancer
}

\author{
XING-YU FENG ${ }^{1,2 *}$, XI-ZHI WEN ${ }^{1,3 *}$, XIAO-JING TAN ${ }^{4,5^{*}}$, JING-HUI HOU $^{1,6}$, YA DING $^{1,3}$, KE-FENG WANG $^{1,3}$, \\ JUN DONG ${ }^{1,3}$, ZHI-WEI ZHOU ${ }^{1,2}$, YING-BO CHEN ${ }^{1,2}$ and XIAO-SHI ZHANG ${ }^{1,3}$ \\ ${ }^{1}$ State Key Laboratory of Oncology in South China, Sun Yat-sen University; ${ }^{2}$ Department of Gastric and Pancreatic Surgery; \\ ${ }^{3}$ Biotherapy Center, Sun Yat-sen University Cancer Center; ${ }^{4}$ Department of Infectious Diseases, The Third Affiliated Hospital; \\ ${ }^{5}$ Hepatology Laboratory, The Hospital for Liver Disease, Sun Yat-sen University; ${ }^{6}$ Department of Pathology, \\ Sun Yat-sen University Cancer Center, Guangzhou, Guangdong 510060, P.R. China
}

Received September 10, 2013; Accepted March 19, 2014

DOI: $10.3892 / \mathrm{mmr} .2014 .2699$

\begin{abstract}
It has been confirmed that B and T lymphocyte attenuator (BTLA; also known as CD272) is a novel co-inhibitory molecule that exhibits a critical role in restraining cell-mediated antitumor immunity. The present study aimed to investigate the expression and prognostic significance of BTLA in gastric adenocarcinoma. Immunohistochemical (IHC) staining was performed to investigate BTLA expression in gastric cancer tissues and normal mucosal tissues. In total, 123 pathologically confirmed specimens were obtained from stage IIIa gastric cancers. A correlation test, Kaplan-Meier curves, and a Cox proportional hazards regression model were used to analyze the data. No BTLA staining in the normal tissues was found, while BTLA-stained gastric carcinoma cells were detected in $75.6 \%(93 / 123)$ of the gastric cancer specimens. High expression levels of BTLA were detected in $31.7 \%(39 / 123)$ of the specimens, while low expression levels were detected in $68.3 \%(84 / 123)$ of the specimens. High BTLA expression levels were associated with shorter survival time, as confirmed by univariate and multivariate analyses. These findings provide a basis for the concept that high BTLA
\end{abstract}

Correspondence to: Professor Ying-Bo Chen, Department of Gastric and Pancreatic Surgery, Sun Yat-Sen University Cancer Center, 651 Dongfeng Road East, Guangzhou, Guangdong 510060, P.R. China

E-mail: chenyb@sysucc.org.cn

Professor Xiao-Shi Zhang, Biotherapy Center, Sun Yat-sen University Cancer Center, 651 Dongfeng Road East, Guangzhou, Guangdong 510060, P.R. China

E-mail: zhangxsh@sysucc.org.cn

*Contributed equally

Key words: B and T lymphocyte attenuator, gastric cancer, survival rate, prognosis expression levels in gastric cancer, identified by IHC, are an independent biomarker for the poor prognosis of patients with gastric cancer.

\section{Introduction}

Gastric cancer was the fourth most common type of malignant tumor worldwide in 2011, with an estimated one million new cases every year (1). More new cases of gastric cancer are diagnosed in China each year than in any other country according to the 2009 Cancer Statistics (2). Although current practice includes incorporating chemotherapy or radiation into surgical resection treatment protocols, gastric cancer survival rates remain poor (3). Several clinicopathological features are reported to be prognostic indicators of gastric cancer. The most important indicator is the stage of the disease. However, in the clinic the prognosis often varies, even among patients with the disease at the same stage $(4,5)$. Therefore, additional prognostic indicators that further characterize the malignant nature of tumors and provide more useful information are urgently required, with the aim of predicting clinical outcomes, individualizing treatments, and identifying molecular targets for those treatments.

The activation of lymphocytes is controlled by two classes of signals: i) Signals triggered by the $\mathrm{T}$ cell receptor upon interaction with antigenic peptides bound to major histocompatibility complex molecules; and ii) signals delivered by the binding of co-receptors to their ligands on antigen-presenting cells (6). The co-receptors include costimulatory and co-inhibitory receptors (7-12). Preclinical and clinical data indicate that the co-inhibitory receptors cytotoxic T lymphocyte-associated protein 4 (CTLA-4) and programmed cell death 1 (PD-1) are responsible for the suppression of human effector $\mathrm{T}$ cell responses to infectious diseases and cancer $(10,11)$, and the therapeutic blockade of these two pathways is currently in clinical development $(13,14)$. Anti CTLA-4 antibody (ipilimumab) was approved by the Food and Drug Administration in March 2011 for treating patients with melanoma that has spread or cannot be removed by surgery (15). Antibody-mediated blockade of 
PD-1 (nivolumab) or PD-L1 (a PD-1 ligand) induced increased clinical response durability of tumor regression and prolonged stabilization of the disease compared with most chemotherapies and kinase inhibitors in patients with advanced cancers, including non-small-cell lung cancer, melanoma and renal cell cancer $(16,17)$. B and T lymphocyte attenuator (BTLA; also known as CD272) is a novel co-inhibitory molecule that is structurally and functionally related to CTLA-4 and PD-1 (18). The ligand of BTLA, herpesvirus entry mediator (HVEM; also known as TNFRSF14), is a member of the other family of co-signaling molecules, the TNF/TNFR superfamily $(19,20)$. BTLA may be a novel target for enhancing antitumor immunity. A study by Derré et al (21) revealed that BTLA is expressed on virus-specific human $\mathrm{CD}^{+} \mathrm{T}$ cells but is progressively downregulated after the cells differentiate from a naive to an effector phenotype. By contrast, tumor-specific human $\mathrm{CD}^{+} \mathrm{T}$ cells continue to express BTLA even after their differentiation into an effector phenotype. Notably, the vaccination of melanoma patients with $\mathrm{CpG}$ led to BTLA downregulation on tumor-specific human $\mathrm{CD}^{+} \mathrm{T}$ cells, concomitant with restoration of the functionality of the cells. Derré et al (21) underscored the therapeutic potential of exploiting the BTLA pathway to treat patients with cancer. Another study revealed that BTLA gene polymorphisms may affect sporadic breast cancer risk and prognosis in Chinese females (22). However, to the best of our knowledge, no previous studies exist concerning the expression status of BTLA in primary gastric cancer, and the prognostic value of BTLA in gastric cancer has not yet been assessed. Thus, the current study detected the expression of BLTA in the primary gastric cancer tissues. The clinical significance of BLTA in the clinical-histological parameters and overall survival of patients with primary gastric cancer was further assessed.

\section{Materials and methods}

Tissue specimens. Formalin-fixed, paraffin-embedded tissues from 123 patients with gastric cancer were used in the present study. Tumor and adjacent normal tissues from the same individual were collected from these patients. Gastric cancer biopsy specimens were collected from patients with stage IIIa (2010 International Union Against Cancer staging system) gastric cancer between 2000 and 2006 at the Sun Yat-sen University Cancer Center (Guangzhou, China) (23). Following removal, the specimen was cut along the opposite side of the tumor. The tumor tissue and the adjacent non-tumor tissue, which were located $>2 \mathrm{~cm}$ apart, were obtained at full thickness. The blocks of specimens were fixed in $10 \%$ formalin for one day and were then trimmed for paraffin blocks. Subsequent to dehydration, hyalinization, paraffin treatment and embedding, 10-16 sheets of serial cross-sections were taken from each block of specimen using a rotary microtome. The thickness of each section was 4-5 nm. The middle sections were stained with hematoxylin and eosin. The rest of the sections were preserved for further examination.

Patients who met the following eligibility criteria were included: i) A diagnosis of gastric adenocarcinoma, identified by histopathological examination; ii) a surgical history that included gastrectomy plus lymphadenectomy (limited or extended); iii) available complete follow-up data; iv) no pre-operative treatment, such as chemotherapy or radiotherapy; v) no history of familial malignancy or other synchronous malignancy (including gastrointestinal stromal tumors, esophageal cancer and colorectal cancer); vi) no recurrent gastric cancer or remnant gastric cancer; and vii) no mortality in the post-operative period due to surgical complications. Tumor resection and D2 lymphadenectomy were performed by experienced surgeons and the surgical procedures, which followed the Japanese Gastric Cancer Association guidelines (24), were similar in all patients who underwent radical resections. Patients received fluorouracil (5-FU)-based adjuvant chemotherapy postoperatively for 6 months. If recurrence or metastasis occurred, 5-FU-based chemotherapy was administered according to the National Comprehensive Cancer Network guidelines (25). This study was conducted in accordance with the Declaration of Helsinki in 2008, and all patients signed a consent form subsequent to being informed that their privacy would be protected. The consent form and the study were approved by the Research Ethics Committee of the Sun Yat-sen University Cancer Center.

IHC and scoring systems. Paraffin-embedded tissues were sectioned continuously at a thickness of $4 \mu \mathrm{m}$ and heated for $1 \mathrm{~h}$ at $65^{\circ} \mathrm{C}$. Briefly, the sections were deparaffinized using xylenes and rehydrated with a graded alcohol series and distilled water. The sections were immersed in an ethylenediamine-NNN'N'-tetraacetic acid antigen retrieval buffer ( $\mathrm{pH} 8.0$ ), subjected to high pressure $(80 \mathrm{kPa})$ for $4 \mathrm{~min}$ for antigen retrieval, and allowed to cool to room temperature. Following blockage with sheep serum, the sections were incubated overnight at $4{ }^{\circ} \mathrm{C}$ with rabbit polyclonal antibody against human BTLA (Abcam, Hong Kong, China), which was diluted to 1:200. Following incubation with secondary antibodies (GBI labs, Mukilteo, WA, USA), the sections were developed using diaminobenzidine tetrahydrochloride and counterstained with hematoxylin. An electron microscope (Leica DM4000 B, Leica, Mannheim, Germany) was used for observation and capturing images. The specimens were analyzed by three independent observers who were blinded to the clinical outcomes of the patients. Discrepancies between the observers were found for $<10 \%$ of the examined slides, and a consensus on each was reached following further review. Total BTLA immunostaining was scored as previously reported (26): The sum of the percent positivity (the percentage of positively stained tumor cells) and the staining intensity. The percent positivity was scored as ' 0 ' $(<5 \%$, negative), ' 1 ' (5-25\%, sporadic), '2' (25-50\%, focal), or '3' ( $>50 \%$, diffuse). The staining intensity was scored as ' 0 ' (no staining), ' 1 ' (weakly stained, visible at high magnification), '2' (moderately stained, visible at low magnification), or ' 3 ' (strongly stained, clearly positive at low magnification). The total BTLA immunostaining score was calculated from the percent positivity score multiplied by the staining intensity score, which resulted in a value of $0-9$. A high BTLA expression level was defined as a total score $\geq 4$, and low BTLA expression level as a total score $<4$.

Follow-up. Postoperative follow-up occurred at the Outpatient Department of Sun Yat-sen University Cancer Center, and included clinical and laboratory examinations every 3 months 

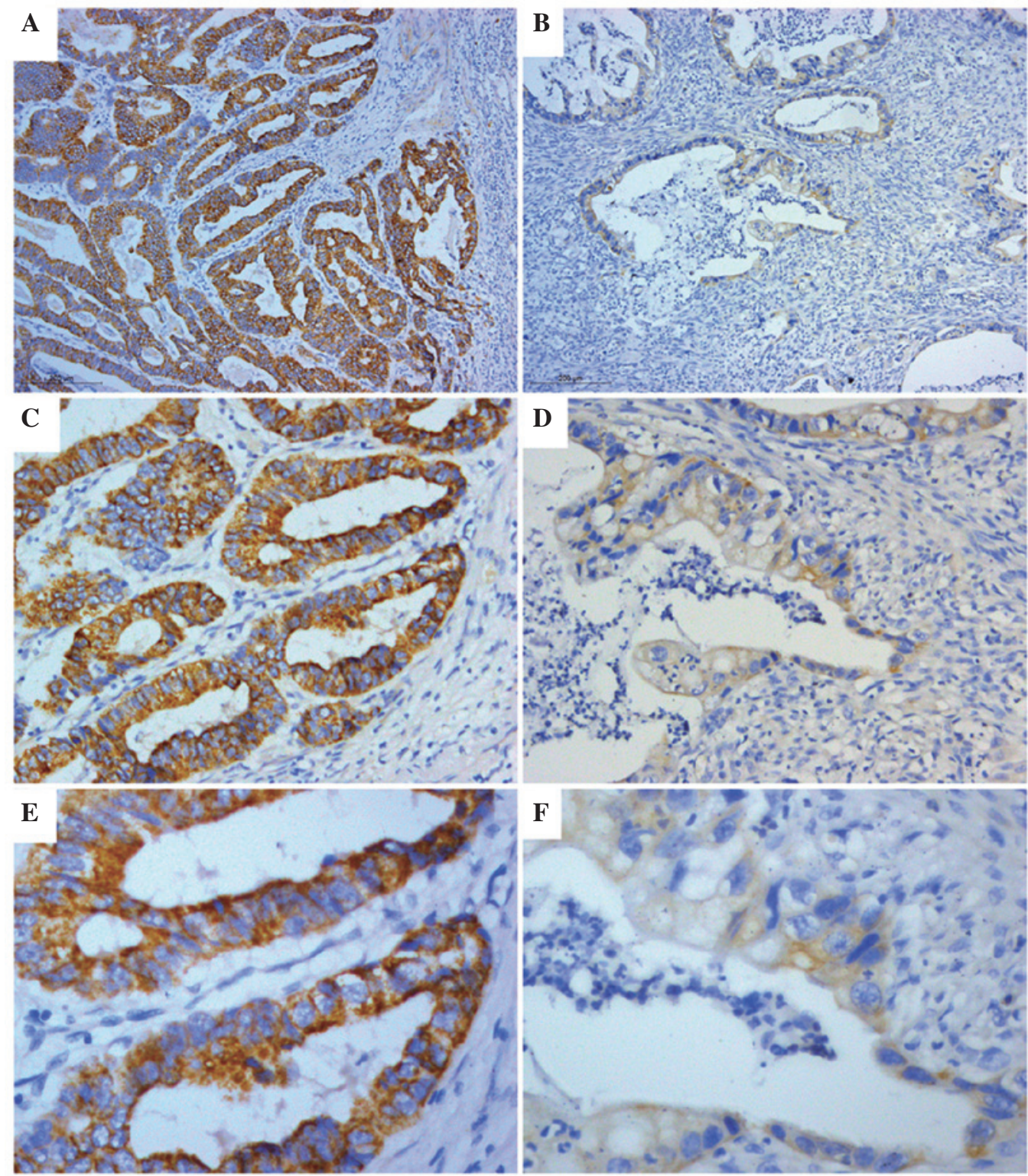

Figure 1. Representative BTLA IHC staining in gastric cancer. (A, C and E) High expression, total percent positivity score $\geq 4$. (B, D and F) Low expression, total percent positivity score <4. Original magnifications: (A and B) x100; (C and D) x200; (E and F) x400. BTLA, B an T lymphocyte attenuator; IHC, immunohistochemical.

for the first 2 years, every 6 months during years 3-5, and annually for an additional 5 years or until patient mortality, depending on the survival time of the patient.

Statistical analysis. All statistical analyses were performed using SPSS statistical software package, version 16.0 (SPSS, Inc., Chicago, IL, USA). The correlations between the expression of BTLA and patient characteristics were analyzed using a correlation test. Kaplan-Meier curves were used to estimate the distribution of variables in relation to survival, which were compared using the log-rank test. Univariate and multivariate analyses were based on the Cox proportional hazards regression model. Overall survival (OS) was defined as time prior to mortality due to any cause, and disease-free survival (DFS) was defined as the time prior to relapse of the primary tumor. $\mathrm{P}<0.05$ was considered to indicate a statistically significant difference.

\section{Results}

Expression patterns of BTLA in gastric tissues. IHC analysis demonstrated that BTLA was highly expressed in a number of cancerous cells of the gastric cancer tissues, whereas there was no BTLA staining observed in the normal tissues. BTLA-stained gastric carcinoma cells were detected in $75.6 \%(93 / 123)$ of the gastric cancer specimens. High expression levels of BTLA were detected in $31.7 \%$ (39/123) of the specimens, while low expression levels were detected in $68.3 \%$ (84/123) of the specimens, which include the 30 samples in which no BTLA staining was detected (Fig. 1).

Correlation between BTLA expression and clinical characteristics of patients. Since BTLA was highly expressed in cancer tissues from a subgroup of the gastric cancer patients, it was determined whether BTLA expression correlates with certain 
Table I. Association between BTLA expression and clinicopathological features of patients with gastric cancer.

BTLA cancer cells

Characteristics

High expression

Low expression

P-value

Gender

Male

0.681

Female

Age (years)

$<60$

$\geq 60$

Tumor location

Proximal

Distant

Whole

Tumor size $(\mathrm{cm})$

$\geq 5$

Histological grade

Well-/moderately differentiated

Lymphatic/venous invasion

\section{No}

Yes

${ }^{\text {aP }}<0.05$ was considered to indicate a statistically significant difference between the high and low BTLA expression groups. ${ }^{\text {bPatients }}$ were staged according to the seventh edition 2010 International Union Against Cancer staging system. BTLA, B and T lymphocyte attenuator.

clinicopathological parameters. As shown in Table I, the expression of BTLA was significantly correlated with the vital status $(\mathrm{P}<0.001)$ and relapse occurrence $(\mathrm{P}=0.002)$ of the patients. In addition, in the high-expression group, BTLA expression was significantly correlated with the tumor location $(\mathrm{P}=0.027)$.

Association between BTLA expression and survival of patients with gastric cancer. The median follow-up time was 49 months, with a range of 4 to 123 months. The cumulative 1-year, 3-year, and 5-year survival rates were 89.4,65.0 and 53.8\%, respectively, for all patients with stage IIIa gastric cancer. The association of
BTLA expression with patient prognosis was evaluated. Patients with low expression levels of BTLA had longer OS $(\mathrm{P}<0.001)$ and DFS $(\mathrm{P}<0.001)$ than those of the patients with high expression levels (Fig. 2). Univariate analysis demonstrated that tumor location, lymphatic/venous invasion and BTLA expression were significant prognostic factors for OS. It was also demonstrated that tumor location and BTLA expression were significant prognostic factors for DFS (Table II).

Multivariate Cox proportional hazards analysis. Since variables observed to have prognostic influence by univariate 
Table II. Univariate analysis of factors associated with OS and DFS.

\begin{tabular}{|c|c|c|c|c|}
\hline \multirow[b]{2}{*}{ Variables } & \multicolumn{2}{|c|}{ OS $(n=123)$} & \multicolumn{2}{|c|}{ DFS $(n=123)$} \\
\hline & HR $(95 \%$ CI $)$ & P-value & $\operatorname{HR}(95 \% \mathrm{CI})$ & P-value \\
\hline Gender & $0.898(0.529-1.527)$ & 0.692 & $0.908(0.547-1.508)$ & 0.710 \\
\hline Age & $1.427(0.871-2.337)$ & 0.158 & $1.321(0.822-2.123)$ & 0.250 \\
\hline Tumor location & $0.388(0.218-0.690)$ & $0.001^{\mathrm{a}}$ & $0.492(0.294-0.823)$ & $0.007^{\mathrm{a}}$ \\
\hline Tumor size (cm) & $1.241(0.755-2.038)$ & 0.395 & $1.143(0.706-1.849)$ & 0.587 \\
\hline Histological grade & $1.075(0.650-1.778)$ & 0.777 & $1.084(0.667-1.760)$ & 0.746 \\
\hline Lymphatic/venous invasion & $2.439(1.103-5.394)$ & $0.028^{\mathrm{a}}$ & $1.914(0.872-4.203)$ & 0.106 \\
\hline Depth of invasion & $1.176(0.761-1.818)$ & 0.465 & $1.230(0.806-1.879)$ & 0.337 \\
\hline Nodal status & $0.850(0.550-1.314)$ & 0.465 & $0.813(0.532-1.241)$ & 0.337 \\
\hline BTLA expression & $4.933(2.908-8.369)$ & $<0.001^{\mathrm{a}}$ & $3.829(2.318-6.325)$ & $<0.001^{\mathrm{a}}$ \\
\hline
\end{tabular}

OS, overall survival; DFS, disease-free survival; HR, hazard ratio; CI, confidence interval; BTLA, B and T lymphocyte attenuator. ${ }^{\text {a }}<0.05$ was considered to indicate a statistically significant difference.

Table III. Multivariate analysis of factors associated with OS and DFS.

\begin{tabular}{|c|c|c|c|c|}
\hline \multirow[b]{2}{*}{ Variables } & \multicolumn{2}{|c|}{ OS $(n=123)$} & \multicolumn{2}{|c|}{ DFS $(n=123)$} \\
\hline & $\mathrm{HR}(95 \% \mathrm{CI})$ & P-value & HR $(95 \% \mathrm{CI})$ & P-value \\
\hline Tumor location & $0.407(0.233-0.710)$ & $0.002^{\mathrm{a}}$ & $0.506(0.308-0.830)$ & $0.007^{\mathrm{a}}$ \\
\hline Lymphatic/venous invasion & $2.961(1.310-6.694)$ & $0.009^{\mathrm{a}}$ & - & - \\
\hline BTLA expression & $5.410(3.125-9.367)$ & $<0.001^{\mathrm{a}}$ & $3.888(2.341-6.459)$ & $<0.001^{\mathrm{a}}$ \\
\hline
\end{tabular}

OS, overall survival; DFS, disease-free survival; HR, hazard ratio; CI, confidence interval; BTLA, B and T lymphocyte attenuator. ${ }^{\text {a }}<0.05$ was considered to indicate a statistically significant difference.
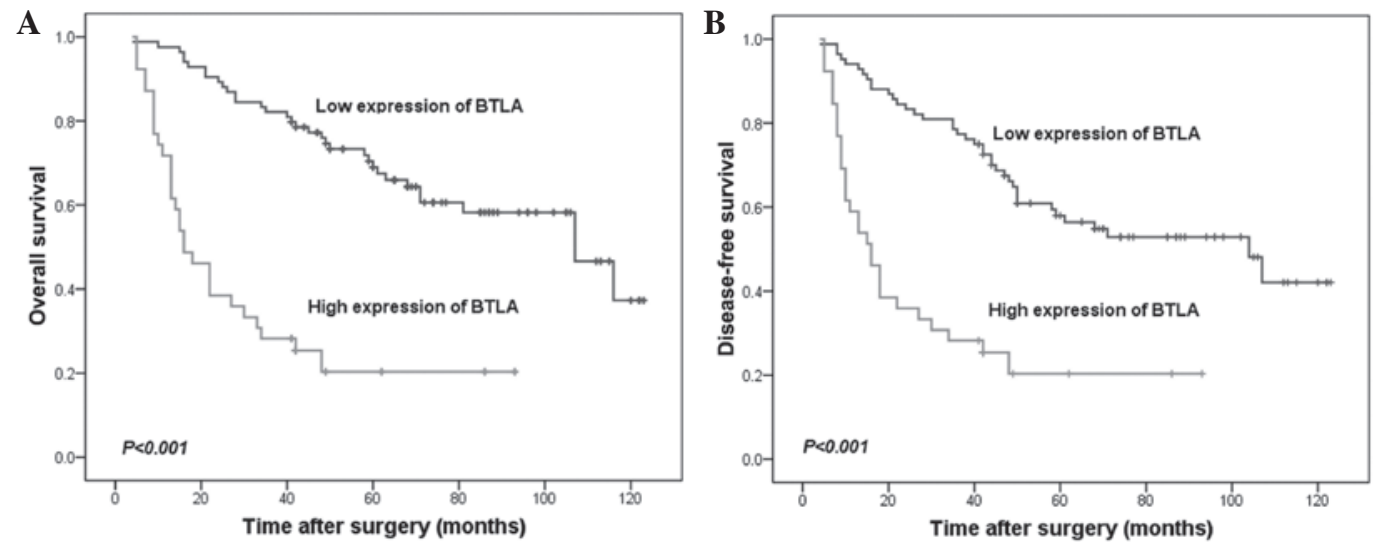

Figure 2. Kaplan-Meier survival analysis of BTLA expression in patients with gastric carcinoma (log-rank test). (A) Correlation of BTLA expression with OS: Low expression, $\mathrm{n}=84$; high expression, $\mathrm{n}=39$. (B) Correlation of BTLA expression with DFS: Low expression, $\mathrm{n}=84$; high expression, $\mathrm{n}=39$. BTLA, B and T lymphocyte attenuator; OS, overall survival; DFS, disease-free survival.

analysis may be covariate, the expression of BTLA and other clinicopathological features that were significantly correlated in the univariate analysis (tumor location and lymphatic/venous invasion) were examined by multivariate analysis. The patients with high BTLA expression levels had significantly reduced OS (HR: 5.410; 95\% CI: 3.125-9.367; $\mathrm{P}<0.001$ ) and DFS (HR: 3.888; 95\% CI: 2.341-6.459; P<0.001) compared with the OS and DFS of the low-expression group (Table III).

\section{Discussion}

BTLA is a novel co-inhibitory molecule that is structurally and functionally related to CTLA-4 and PD-1. Gavrieli et al (27) 
reported BTLA expression on T cells, B cells, dendritic cells and myeloid cells. Derré et al (21) reported that naive human $\mathrm{CD}^{+} \mathrm{T}$ cells express high levels of BTLA on their cell surface. Thus far, several co-inhibitory molecules have been analyzed in human solid tumor-derived cells, including CTLA-4 and TIM-3 (28-31), but no results are available regarding the expression of BTLA on this type of tumor cell. To the best of our knowledge, the current study is the first to confirm that BTLA can be constitutively detected in primary gastric carcinomas using IHC. In the present study, BTLA-stained gastric carcinoma cells were detected in $75.6 \%(93 / 123)$ of gastric cancer specimens. However, BTLA was not expressed in normal tissues. This result preliminarily indicated that the expression of BTLA is closely associated with the progression of gastric cancer.

Furthermore, this retrospective study represents the first investigation of BTLA expression as a possible prognostic factor for DFS and OS in patients with radically resected stage IIIa gastric cancer to the best of our knowledge. Several studies have found that the prognosis often varies, even among patients with the same disease stage. Therefore, additional prognostic indicators that could further characterize the malignant nature of the tumors are urgently required to provide more useful information $(4,5,32)$. In the current study, all patients had stage IIIa cancer, but patients with high BTLA expression levels had shorter DFS and OS than those of the patients with low expression levels of BTLA. Notably, Cox multivariate analysis demonstrated that the expression of BTLA within cancer tissue was an independent prognostic factor. These data indicated that BTLA acts in the progression of gastric cancer. We hypothesize that the overexpression of BTLA leads to a poorer prognosis due to greater downregulation of $\mathrm{T}$ cell activation. Fourcade et al (33) previously demonstrated that upregulation of BTLA and PD-1 is involved in restricting NY-ESO-1-specific $\mathrm{CD}^{+} \mathrm{T}$ cell expansion and function in melanoma. These cells were partially dysfunctional, producing fewer IFN $\gamma$ than BTLA- T cells. BTLA blockade enhanced the expansion, proliferation, and cytokine production of NY-ESO-1-specific $\mathrm{CD}^{+} \mathrm{T}$ cells. Together, these results suggest that high levels of BTLA expression on TA-specific $\mathrm{CD}^{+} \mathrm{T}$ cells and upregulation of HVEM on tumor cells may be another inhibitory pathway developed by cancer cells to impair the antitumor immune response. Notably, Pasero et al (34) showed that BTLA is also implicated in the homeostatic regulation of $\mathrm{V} \gamma 9 \mathrm{~V} \delta 2 \mathrm{~T}$ cells. A blockade of the BTLA-HVEM interaction allowed improved spontaneous or $\mathrm{T}$ cell receptor-induced proliferation of allogeneic and autologous $\gamma \delta$ T cells in co-culture with $\mathrm{HVEM}^{+}$lymphoma cells. Thus, in addition to immune escape from 'conventional' T lymphocytes, a BTLA-HVEM inhibitory interaction may represent a pathway for tumor cells to evade $\gamma \delta$ T cell recognition. The blockade of this pathway may restore the recognition and the efficacy of T lymphocytes and $\gamma \delta \mathrm{T}$ cells.

Furthermore, it has been reported that BTLA is a valid target for cancer immunotherapy (21). The co-inhibitory molecule BTLA can inhibit tumor-specific human $\mathrm{CD}^{+} \mathrm{T}$ cells, and vaccination with $\mathrm{CpG}$ adjuvants at least partly overcomes this barrier by downregulating BTLA. CpG-mediated downregulation of BTLA correlates with restoration of the in vivo effector function of tumor-specific human $\mathrm{CD} 8^{+} \mathrm{T}$ cells (21).
These data underscore the therapeutic potential of exploiting the BTLA pathway to treat patients with cancer. In the present study, expression of BTLA was detected in 75.6\% (93/123) of the gastric cancer patients. Thus, blocking the BTLA pathway may be a novel method for treating gastric cancer.

In conclusion, the current study demonstrated the expression of BTLA in tumor cells from patients with gastric cancer using IHC for the first time, to the best of our knowledge. Most notably, the univariate and multivariate analyses revealed the significant role of BTLA as an independent prognostic factor in patients with gastric cancer. The status of BTLA expression may be determined by clinical examination and immunohistochemical analysis.

\section{Acknowledgements}

The authors thank Professor Xiao-mou Peng of the Hospital for Liver Disease of Sun Yat-sen University for providing laboratory equipment support. Funding for this study was provided by the Science and Technology Projects of Guangdong Province (2011B061300052) and the National Natural Science Foundation of China (81272341).

\section{References}

1. Jemal A, Bray F, Center MM, Ferlay J, Ward E and Forman D: Global cancer statistics. CA Cancer J Clin 61: 69-90, 2011.

2. Jemal A, Siegel R, Ward E, Hao Y, Xu J and Thun MJ: Cancer statistics, 2009. CA Cancer J Clin 59: 225-249, 2009.

3. Ajani JA, Barthel JS, Bekaii-Saab T, et al; NCCN Gastric Cancer Panel: Gastric cancer. J Natl Compr Canc Netw 8: 378-409, 2010

4. Park do J, Kong SH, Lee HJ, et al: Subclassification of pT2 gastric adenocarcinoma according to depth of invasion (pT2a vs pT2b) and lymph node status (pN). Surgery 141: 757-763, 2007.

5. Liu X, Xu Y, Long Z, Zhu H and Wang Y: Prognostic significance of tumor size in T3 gastric cancer. Ann Surg Oncol 16: 1875-1882, 2009

6. Bretscher PA: A two-step, two-signal model for the primary activation of precursor helper T cells. Proc Natl Acad Sci USA 96: 185-190, 1999.

7. Chambers CA and Allison JP: Costimulatory regulation of T cell function. Curr Opin Cell Biol 11: 203-210, 1999.

8. Chambers CA, Kuhns MS, Egen JG and Allison JP: CTLA-4-mediated inhibition in regulation of T cell responses: mechanisms and manipulation in tumor immunotherapy. Annu Rev Immunol 19: 565-594, 2001.

9. Barber DL, Wherry EJ, Masopust D, et al: Restoring function in exhausted CD8 T cells during chronic viral infection. Nature 439: 682-687, 2006.

10. Peggs KS, Quezada SA and Allison JP: Cell intrinsic mechanisms of T-cell inhibition and application to cancer therapy. Immunol Rev 224: 141-165, 2008.

11. Ha SJ, West EE, Araki K, Smith KA and Ahmed R: Manipulating both the inhibitory and stimulatory immune system towards the success of therapeutic vaccination against chronic viral infections. Immunol Rev 223: 317-333, 2008.

12. Blackburn SD, Shin $\mathrm{H}$, Haining WN, et al: Coregulation of $\mathrm{CD}^{+}$ $\mathrm{T}$ cell exhaustion by multiple inhibitory receptors during chronic viral infection. Nat Immunol 10: 29-37, 2009.

13. Topalian SL, Hodi FS, Brahmer JR, et al: Safety, activity, and immune correlates of anti-PD-1 antibody in cancer. N Engl J Med 366: 2443-2454, 2012.

14. Hodi FS, O'Day SJ, McDermott DF, et al: Improved survival with ipilimumab in patients with metastatic melanoma. N Engl J Med 363: 711-723, 2010.

15. Eggermont AM and Robert C: New drugs in melanoma: it's a whole new world. Eur J Cancer 47: 2150-2157, 2011.

16. Brahmer JR, Tykodi SS, Chow LQ, et al: Safety and activity of anti-PD-L1 antibody in patients with advanced cancer. N Engl J Med 366: 2455-2465, 2012. 
17. Wolchok JD, Kluger H, Callahan MK, et al: Nivolumab plus ipilimumab in advanced melanoma. N Engl J Med 369: 122-133, 2013.

18. Watanabe N, Gavrieli M, Sedy JR, et al: BTLA is a lymphocyte inhibitory receptor with similarities to CTLA-4 and PD-1. Nat Immunol 4: 670-679, 2003.

19. Sedy JR, Gavrieli M, Potter KG, et al: B and T lymphocyte attenuator regulates $\mathrm{T}$ cell activation through interaction with herpesvirus entry mediator. Nat Immunol 6: 90-98, 2005.

20. Gonzalez LC, Loyet KM, Calemine-Fenaux J, et al: A coreceptor interaction between the CD28 and TNF receptor family members $\mathrm{B}$ and T lymphocyte attenuator and herpesvirus entry mediator. Proc Natl Acad Sci USA 102: 1116-1121, 2005.

21. Derré L, Rivals JP, Jandus C, et al: BTLA mediates inhibition of human tumor-specific $\mathrm{CD} 8^{+} \mathrm{T}$ cells that can be partially reversed by vaccination. J Clin Invest 120: 157-167, 2010.

22. Fu Z, Li D, Jiang W, et al: Association of BTLA gene polymorphisms with the risk of malignant breast cancer in Chinese women of Heilongjiang Province. Breast Cancer Res Treat 120: 195-202, 2010

23. Sobin LH, Gospodarowicz MK and Wittekind C: International Union Against Cancer (UICC) TNM classification of malignant tumours. 7. New York: Wiley-Liss, 2010.

24. Japanese Gastric Cancer Association: Japanese Classification of Gastric Carcinoma - 2nd English Edition. Gastric Cancer 1: 10-24, 1998.

25. Ajani JA, Barthel JS, Bekaii-Saab T, et al; NCCN Gastric Cancer Panel: Gastric cancer. J Natl Compr Canc Netw 8 : 378-409, 2010.
26. Wang W, Lv L, Pan K, et al: Reduced expression of transcription factor AP- $2 \alpha$ is associated with gastric adenocarcinoma prognosis. PLoS One 6: e24897, 2011.

27. Gavrieli M, Sedy J, Nelson CA and Murphy KM: BTLA and HVEM cross talk regulates inhibition and costimulation. Adv Immunol 92: 157-185, 2006.

28. Salvi S, Fontana V, Boccardo S, et al: Evaluation of CTLA-4 expression and relevance as a novel prognostic factor in patients with non-small cell lung cancer. Cancer Immunol Immunother 61: 1463-1472, 2012.

29. Contardi E, Palmisano GL, Tazzari PL, et al: CTLA-4 is constitutively expressed on tumor cells and can trigger apoptosis upon ligand interaction. Int J Cancer 117: 538-550, 2005.

30. Mao H, Zhang L, Yang Y, et al: New insights of CTLA-4 into its biological function in breast cancer. Curr Cancer Drug Targets 10: 728-736, 2010.

31. Zhuang X, Zhang X, Xia X, et al: Ectopic expression of TIM-3 in lung cancers: a potential independent prognostic factor for patients with NSCLC. Am J Clin Pathol 137: 978-985, 2012.

32. Dong J, Li J, Liu SM, et al: CD $33^{+} /$p-STAT1 $^{+}$double-positive cell as a prognostic factor for stage IIIa gastric cancer. Med Oncol 30: 442,2013

33. Fourcade J, Sun Z, Pagliano O, et al: CD8(+) T cells specific for tumor antigens can be rendered dysfunctional by the tumor microenvironment through upregulation of the inhibitory receptors BTLA and PD-1. Cancer Res 72: 887-896, 2012.

34. Pasero C and Olive D: Interfering with coinhibitory molecules: BTLA/HVEM as new targets to enhance anti-tumor immunity. Immunol Lett 151: 71-75, 2013. 